\title{
Association of high adherence to vegetables-and- fruits dietary pattern with quality of life among Chinese women with early stage breast cancer
}

\section{Yuan-Yuan Lei}

Chinese University of Hong Kong

Suzanne C. Ho

Chinese University of Hong Kong

Carol Kwok

Princess Margaret Hospital Cancer Centre

Ashley Cheng

Princess Margaret Hospital Cancer Centre

\section{Ka Li Cheung}

Chinese University of Hong Kong

\section{Roselle Lee}

Chinese University of Hong Kong

\section{Frankie Mo}

Chinese University of Hong Kong

Winnie Yeo ( $\nabla$ winnieyeo@cuhk.edu.hk)

Chinese University of Hong Kong

\section{Research Article}

Keywords: breast cancer, dietary pattern, quality of life, Chinese women

Posted Date: March 26th, 2021

DOl: https://doi.org/10.21203/rs.3.rs-331727/v1

License: (c) (1) This work is licensed under a Creative Commons Attribution 4.0 International License. Read Full License

Version of Record: A version of this preprint was published at Quality of Life Research on September 16th, 2021. See the published version at https://doi.org/10.1007/s11136-021-02985-0. 


\section{Abstract}

Purpose: Dietary intake and patients' quality of life $(\mathrm{Q} o \mathrm{~L})$ are important supportive care issues in breast cancer survivorship. This study aimed to identify dietary pattern after breast cancer diagnosis. In addition, the association between dietary patterns and QoL were cross-sectionally investigated.

Methods: A breast cancer cohort which included 1462 Chinese women were longitudinally interviewed at four time-points, namely baseline, 18-, 36-, and 60-months after diagnosis. At each follow-up, validated food frequency questionnaires (FFQ) were used to assess patients' dietary intake, and principal component analysis was used to derive dietary patterns. European Organization for Research and Treatment of Cancer Quality of Life Questionnaire Core 30 (EORTC QLQ-C30) were used to measure QoL. This study included 1226 patients with invasive disease who completed assessment at 18-month followup and had detailed data of dietary intake and QoL.

Results: Two major dietary patterns were identified: "grain and animal food pattern" and "vegetables and fruits pattern". Multivariable linear regression analysis indicated that grain and animal food intake was inversely associated with score for fatigue $(P=0.010)$ and dyspnea $(P=0.024)$. Vegetables and fruits intake were positively associated with scores for global health status/QoL $(P=0.002)$, while inversely associated with score for loss of appetite $(P=0.040)$ and diarrhea $(P=0.009)$.

Conclusion: This study suggested that high adherence to a vegetables and fruits pattern might have beneficial effect on QoL among Chinese breast cancer patients. Prospective follow-up data could further confirm whether a specific dietary pattern has impact on cancer outcomes.

\section{Plain English Summary}

In recent decades, dietary intake and patients' quality of life (QoL) are important supportive care issues in breast cancer survivorship. This study was based on prospective cohort study, which enrolled 1462 Chinese women with early-stage breast cancer. In the present analysis, it first identified the post-diagnosis dietary pattern, and then cross-sectionally investigated the associations between dietary patterns and QoL at 18-month follow-up. Two major dietary patterns were identified by factor analysis, termed "grain and animal food pattern" and "vegetables and fruits pattern". The association between "grain and animal food pattern" and QoL showed that "grain and animal food pattern" was inversely associated with fatigue and dyspnea. The association between "vegetables and fruits pattern" and QoL showed that vegetables and fruits food intake was positively associated with scores for global health status/QoL, but inversely associated with scores in loss of appetite and diarrhea. The present study suggested a potential beneficial effect on QoL for breast cancer patients following a healthy lifestyle habit. Therefore, interventions to encourage breast cancer survivors to adopt a healthy dietary habit should be pursued.

\section{Introduction}


Hong Kong is a relatively more westernized and urbanized city in China. Breast cancer is the most common cancer and the third leading cause of cancer-related mortality in local females.[1] During the three decades between 1976 and 2010, the age-standardized breast cancer incidence rate have increased on average by $1.69 \%$ per year, while the mortality rates have decreased on average by $0.02 \%$ per year.[2] Epidemiology study estimated that this trend would continue in the next 15 years. Under such circumstances, the population of breast cancer survivors in Hong Kong would grow substantially.[2] While the improvement of breast cancer survival has been encouraging, a critical new issue is in great need of attention; that is, survivorship of the growing population of breast cancer patients, with dietary intake and quality of life (QoL) both being important aspects to be addressed.

Several cross-sectional studies have investigated the association between specific food item and QoL in breast cancer survivors. A Chinese study reported that higher vegetables and fruits intake were significantly associated with better QoL in breast cancer survivors.[3] Blanchard et al. also assessed the association between fruits and vegetables intake and QoL among cancer survivors in the US.[4] Patients were defined as meeting the American Cancer Society (ACS) 5-A-Day recommendation if they consumed at least 5 servings of fruits and vegetables per day. The results showed that breast cancer survivors who met the 5-A-Day recommendation had slightly yet significantly higher QoL compared with those who did not.[4]

Given food items are consumed in combination and their complex effects are likely to be interactive or synergistic, the focus of nutrition research has shifted from specific food item or nutrient to the overall diet and diet pattern in recent years.[5; 6] Dietary pattern analysis investigated the effect of the overall diet by integrating a comprehensive food or nutrients intake. $[5 ; 6]$ Three cohort studies have investigated the association between dietary pattern and breast cancer outcome.[7-9] Two of them were conducted in US women, and similar dietary patterns were identified: "Prudent dietary pattern" (characterized by higher intake of fruits, vegetables, whole grains) and "Western dietary pattern" (characterized by higher intake of refined grains, processed meat, red meat). The results showed that higher adherence to the "Prudent dietary pattern" and lower adherence to the "Western dietary pattern" might protect against mortality from causes unrelated to breast cancer.[7; 8] Another study was undertaken in German post-menopausal women with breast cancer. The "healthy dietary pattern", namely high consumption of vegetables, fruits, vegetable oil, sauces/condiments, and soups/bouillons, was inversely associated with overall mortality and breast cancer recurrence among patients with stage I-IIIA. On the other hand, higher consumption of "unhealthy dietary pattern" was associated with an increased risk of non-breast cancer mortality.[9] While the effect of dietary pattern on QoL has also been explored, the evidence on potential association has been limited. Only a Korean study has explored such association in women with breast cancer.[10] Up to now, the association between post-diagnosis dietary pattern and breast cancer survival or QoL has not been reported in Chinese women.

Based on a prospective breast cancer cohort, this study aimed to first identify the post-diagnosis dietary pattern, and then cross-sectionally investigate the associations between dietary patterns and QoL at 18month follow-up. 


\section{Methods}

\section{Patients}

A prospective cohort study named the Hong Kong NTEC-KWC Breast Cancer Survival Study (HKNKBCSS) was initiated in 2011. This enrolled Chinese women with early stage breast cancer, and aimed to investigate whether soy isoflavones and other lifestyle factors were related to breast cancer outcomes. [11-14] The eligibility criteria were defined as follow: histologically confirmed breast cancer with American joint Committee on Cancer (AJCC) stage 0-III diagnosed within 1 year before study entry,[15] any age, female gender, Chinese ethnicity, able to read Chinese, and had no prior history of breast or other cancers.

In total, the study cohort recruited 1462 patients from two regional cancer centers in Hong Kong. Each participant provided written informed consent. Enrolled patients were interviewed at four time-points: baseline at study entry, 18-month, 36-month and 60-month follow-up post-diagnosis. The study was approved by the Joint CUHK-NTEC Clinical Research Ethics Committee and the KWC Research Ethics Committee of the Chinese University of Hong Kong and the Hong Kong Hospital Authority.

The 18-month follow-up was conducted between 12 and 24 months after breast cancer diagnosis, during which 1310 patients in the study cohort completed assessment at that time-point. Participants who had incompletely filled questionnaire for $\mathrm{QoL}(\mathrm{n}=4)$, who reported implausible dietary intake (energy intake estimates $<500$ or $>4000$ kcal per day; $n=3)$ or who had carcinoma in-situ $(n=77)$ were excluded, resulting in 1226 patients for the present analysis.

\section{Data collection at each assessment}

Trained personnel conducted face-to-face interview at baseline and each follow-up. During each interview, socio-demographic data were collected by structured questionnaires, and included age, marital status, occupation, education level, family income, menopausal status and prior medical history. Validated questionnaires were used to collect information about lifestyle factors, such as dietary intake and level of physical activity. At each assessment, body weight and height were also measured. Body mass index (BMI) classification was based on criteria adopted in the Asia-Pacific region, which consisted of four groups, as follow: underweight $<18.5 \mathrm{~kg} / \mathrm{m}^{2}$, normal $18.5-22.9 \mathrm{~kg} / \mathrm{m}^{2}$, overweight $23-24.9 \mathrm{~kg} / \mathrm{m}^{2}$ and obese $\geq 25 \mathrm{~kg} / \mathrm{m}^{2}$.[16] Patients' clinical information on breast cancer was retrieved by reviewing hospital medical records.

\section{Dietary intake and dietary pattern assessment}

Patients recalled their dietary intake over the proceeding 12 months at 18-month follow-up assessment. Dietary intake was collected by filling a validated food frequency questionnaires (FFQ) during face-to-face interview.[17] The FFQ contained 109 food items that were commonly consumed in Hong Kong population and a specific proper portion size was used for quantification of each food item. Based on the design of FFQ, patients should report the frequency of consumption and average amount of intake at 
each time for each food item. Interviewers would provide a food photographs with individual food portions during the interview, which was useful for more clear estimation. Based on data collected on FFQ, the daily total energy intake and other nutrients can be calculated according to Chinese Food Composition Table.[18]

After excluding uncommon dietary items (with average intake $<2$ serving/month), the 109 food items in the FFQ were grouped into 17 food groups based on the similarity of food type, nutrient profiles as well as local eating habits in local Chinese population (Supplementary table 1). This approach has also been adopted by previous studies, and it could reduce the complexity of dietary data.[6-8] Based on the daily consumption of the 17 food groups that was not energy adjustment, principal component analysis was used to derive dietary patterns. The food groups (factors) were rotated by orthogonal transformation (Varimax rotation function), resulting in uncorrelated, independent factors. Major factors retained were based on eigenvalue greater than 1.0, the scree plot (supplementary figure 1), and factor interpretability, which were commonly used in breast cancer studies.[19]

Factor loadings represent correlation coefficients between the food groups and the dietary pattern. The derived factors (patterns) were labeled as major food groups with higher factor loading as well as the interpretation of the data. This study identified two primary factors, which were labeled "grain and animal food pattern" and "vegetables and fruits pattern". The factor score for each dietary pattern was calculated for each participant by summing intakes of food groups weighted by their factor loading.[6] Each individual was assigned a score for each identified dietary pattern, which reflect one's conformity with that pattern; a higher factor score suggested better conformity with that pattern.

\section{QoL assessment using EORTC QLQ-C30}

EORTC QLQ-C30 was used to measure patients' QoL at the time of 18-month follow-up assessment.[20] EORTC QLQ-C30 was designed to assess a range of cancer-specific QoL issues relevant to a broad spectrum of cancer patients.[21] This questionnaire consisted of 30 cancer-specific questions with multiple-point scales, including a global health status/QoL scale, five functional scales (physical, role, emotional, cognitive and social), nine symptom scales (fatigue, nausea and vomiting and pain, dyspnea, insomnia, appetite loss, constipation, diarrhea and financial difficulty). According to the EORTC QLQ C-30 Scoring Manual, multiple-point scales were transformed into standard scores (from 0 to 100) in the analysis. High scores on global health status/QoL and functioning scales represented good QoL, while high scores on the symptom scales indicated more severe symptoms.[22]

\section{Statistical analysis}

For comparing the characteristics of participants, they were categorized into three groups (tertile 1, tertile 2 and tertile 3 ) according to the tertiles of factor scores in each dietary pattern. Analysis of variance was used for continuous data and chi-square test for categorical data. Multivariable linear regression models were used to investigate the association between dietary pattern and QoL items. QoL items were $\log _{10}$ transformed to fulfil the normal distribution assumption. The potential confounders were introduced in 
models using the enter method, which were identified based on the theoretical considerations, the previous literature, and the results of univariate analyses. Collinearity diagnostics were applied to exclude multicollinearity of variables. The following covariables were adjusted in the first model age at 18-month follow-up (years; continuous), education level (high school or below, college or above), household income $(<15,000,15,000-30,000, \geq 30,000 \mathrm{HKD} /$ month), total number of comorbidities $(0,1, \geq 2)$, menopausal status at 18-month follow-up (pre-menopausal, post-menopausal), AJCC stage (I, II, III, I-III without detail), ER status (positive, negative, missing), PR status (positive, negative, missing), current adjuvant hormonal therapy usage (yes, no), BMl at 18-month follow-up ( $\mathrm{kg} / \mathrm{m} 2$; continuous), level of physical activity (METhours/week; continuous), total and energy intake (kcal/day; continuous). In the second model, factor scores of the other dietary pattern (when one of the two dietary patterns were being analyzed; continuous) were further adjusted. All analyses were performed using SPSS 26.0; and $P$ value $<0.05$ based on twosided analysis were considered statistically significant.

\section{Results}

\section{Two major dietary patterns}

Two major dietary patterns were identified by factor analysis. The representative food groups with their factor loadings in the dietary pattern were shown in Table 1. The first, termed "grain and animal food pattern", was characterized by high factor loadings for refined grain, red meat, fish and seafood, oil, cakes and snacks, processed meat, eggs. The second, termed "vegetables and fruits pattern", was characterized by high factor loadings for leaf vegetables, other vegetables, potato, fruits and legumes. These two factors explained $38.38 \%$ of the total variance $(26.23 \%$ and $12.15 \%$, respectively). Further sensitivity analyses excluding those patients who were recruited more than 6 months $(n=153)$ after diagnosis were also conducted.

\section{Patients' characteristics according to the tertiles of factor score in each dietary pattern}

Patients' characteristics were compared according to the tertiles of factor score in each dietary pattern, and the results were summarized in Table 2. Patients in the highest tertile of "grain and animal food pattern" were younger, more likely to be pre-menopausal and had higher energy intake. In addition, compared to women in the lowest and highest tertile of "grain and animal food pattern", the proportion of patients who were married was higher in the second tertiles. Patients in the highest tertile of "vegetables and fruits pattern" attained higher education level, were more likely to have normal BMI, were more physically active and had higher energy intake. Additionally, patients grouped into the second tertile factor score of "vegetables and fruits pattern" were more likely to be married, ER positive, PR positive and had higher household income.

\section{The association between "grain and animal food pattern" and QoL}

Multivariable linear regressions (Table 3 ) showed that after controlling for the potential confounders, "grain and animal food pattern" was inversely associated with fatigue $(P=0.010)$ and dyspnea $(P=$ 
0.024). An increase of 1 SD of "grain and animal food pattern" score was associated with a decrease of fatigue score by 0.133 and a decrease of dyspnea score by 0.117 .

\section{The association between "vegetables and fruits pattern" and QoL}

Multivariable linear regressions (Table 4) indicated that after controlling for the potential confounders, vegetables and fruits food intake was positively associated with scores for global health status/QoL ( $P=$ $0.002)$, but inversely associated with scores in loss of appetite $(P=0.040)$ and diarrhea $(P=0.009)$. An increase of 1 SD of "vegetables and fruits pattern" score was associated with an increase of global health status/QoL score by 0.118 ; while an increase of $1 \mathrm{SD}$ of "vegetables and fruits pattern" score was associated with a decrease of loss of appetite score by 0.081 and a decrease of diarrhea score by 0.102 .

\section{Discussion}

Using data assessed at 18-month follow-up from a prospective cohort of Chinese women with breast cancer, two dietary patterns were identified, namely "grain and animal food pattern" and "vegetables and fruits pattern". Women with a higher consumption of vegetables and fruits tended to have better scores for global health status/QoL as well as less symptoms in diarrhea.

The relationship between dietary pattern and QoL has only been investigated in a cross-sectional study among 232 Korean breast cancer survivors. This study used 3-day dietary record to assess dietary intake, in which two major dietary patterns were identified, "healthy dietary pattern" and "western dietary pattern". [23] Higher "healthy dietary pattern" score was associated with milder dyspnea while more severe symptom of insomnia. Of note, even though the 3-day dietary record was regarded as a gold standard to validate FFQ, they cannot comprehensively represent the habitual dietary intake over a relative long time. The dietary patterns identified and their associations with QoL were different with the present results, which may reflect the different dietary habits in Hong Kong and Korea. A few studies have evaluated the impact of quality of diet on QoL in breast cancer survivors. In the Health, Eating, Activity and Lifestyle (HEAL) study, dietary intake was assessed by FFQ, and those dietary data was used to evaluate diet quality by the Diet Quality Index (DQI).[24] The DQI assessed an individual's adherence to eight dietary recommendations from the National Academy of Sciences regarding fat, saturated fat, cholesterol, sodium, fruits/vegetables, grain, protein and calcium intake. According to the level of compliance, the diet quality scores can be categorized into four groups: excellent, good, fair and poor. Compared to women with poor diet quality, those with excellent diet quality had significantly higher Mental component summary (MCS) scores as evaluated by Medical Outcomes Study Short Form Survey 36 (SF-36).[24] In another report from the cohort of the HEAL study, diet quality was evaluated by the Healthy Eating Index 2010 (HEI-2010).[25] Based on their dietary data, a HEI-2010 score ranging from 0 to 100 points was calculated for each patient. According to the HEI-2010 score, patients were classified into quartiles (Q4 to Q1, from better-quality to poor-quality). The results showed that higher quality of diet was associated with reduced cancer-related fatigue.[25] Mosher et al. reported the association between diet and QoL among elderly cancer survivors, including those with breast cancer.[26] Overall diet quality was assessed on two 
days based on 24-hour recalls, and subsequently evaluated by HEI 2005 (HEl-2005). Each participant had a HEl-2005 score, ranging from 0 (worst) to 100 (best). Diet quality was positively related to Physical component summary (PCS) score as measured by SF-36.[26]

The present study found that patients with higher intake of grain and animal food were younger and more likely to be pre-menopausal. These findings contributed to our understanding of breast cancer patients' dietary habits, whereby healthcare professionals should pay more attention to educate younger patients to limit the consumption of red meat and processed meat. In addition, patients with higher intake of vegetables and fruits had higher education level, were more likely to have normal BMI and be more physically active. It reflected that patients who had higher education level were more likely to adopt a healthier dietary habit. Furthermore, patients who cared about their diet also tend to adopt a healthier lifestyle habit, being more physically active and more likely to maintain a normal weight.

The findings suggested that higher adherence to "grain and animal food pattern" may be inversely related to fatigue and dyspnea. These associations have not been reported in previous studies and the potential mechanisms underlying such associations were not clear. Of note, patients with higher intake of grain and animal food were younger; and younger patients have reported less fatigue [27] and dyspnea [28] than older ones. Hence, these associations may be influenced by patients' age, although age at 18-month follow-up have been adjusted in the multivariable analyses.

The current study suggested higher adherence to "vegetables and fruits pattern" was associated with better global health status/QoL and milder symptoms of loss of appetite and diarrhea. Such associations were consistent with a previous cross-sectional study in Chinese women with breast cancer, which included 3,344 women with most patients (63\%) having survived for at least 5 years.[3] Participants were asked to report the frequency of vegetables and fruits intake in the past month and they underwent QoL assessment at the same time. EORTC QLQ-C30 was used to measure QoL. Compared to women who had vegetables $\leq 250 \mathrm{gram} /$ day, breast cancer survivors who consumed vegetables $>250 \mathrm{gram} /$ day had significantly higher scores in most QoL dimensions, including global health status/QoL, physical, cognitive and emotional functioning, as well as milder symptoms of dyspnea, insomnia, loss of appetite and constipation. In addition, compared to women who did not eat fruits every day, those who had fruits every day reported significantly higher scores in all QoL dimensions, except for symptom items.[3] The association between fruits and vegetables intake and QoL among cancer survivors has also been investigated in the US women.[4] Patients were defined as meeting the ACS 5-A-Day recommendation if they consumed at least 5 servings of fruits and vegetables per day. Results showed that breast cancer survivors who met the 5-A-Day recommendation had slightly yet significantly higher overall global health composite score compared with those who did not.[4] The potential mechanism underlying the association between specific dietary pattern or food item with QoL has not been fully elucidated. One mechanism may underlie the association between dietary intake and gastrointestinal symptoms: vegetables and fruits contain high quantities of dietary fiber which might improve gastrointestinal function, and relief constipation and diarrhea.[29] 
Based on data from a prospective cohort study with large sample size, this was the first study to investigate the associations between dietary patterns and QoL among Chinese breast cancer survivors. In addition, this study used a validated FFQ to measure dietary intake, which allowed the collection of patients' dietary intake information over the previous year in a relatively accurate manner. One of the major limitations of the present study was that as in other dietary pattern studies, principal component analysis used to identify dietary patterns involves some arbitrary decisions, including grouping of food items, method of factor rotation and labeling of dietary pattern.[30] In addition, the two retained components explained $38.38 \%$ of the item variance, and it might not adequately represent the food consumption pattern. Furthermore, the relationship of diet pattern and QoL was only examined at one time-point, further studies in other time-points are needed. Of note, the 18-month follow-up assessment was an important time-point, in which patients' dietary intakes during the previous year, namely from 6 to 18 months post-diagnosis were collected. During this period, breast patients have just completed intense anti-cancer therapy, including surgery, radiotherapy and/or chemotherapy. Hence, it is a potential pivotal turning period for a cancer survivor to adopt changes in lifestyle. In fact, earlier analysis of dietary change in this cohort has suggested that breast cancer survivors made notable dietary change at the time of diagnosis or soon thereafter, and such changes tended to be sustained in the following survivorship.[11] Last but not the least, the current study is of exploratory rather than confirmatory nature. As such, the positive effects of a vegetable-based diet on QoL outcomes in breast cancer patients reported in the present study should be further supported by confirmatory analyses with preregistered hypothesis and analysis protocols in future research.

Of note, cultural background of the dietary patterns should be considered in the interpretation of the results. For instance, higher intake of "vegetables and fruit pattern" tends to be a part of traditional Chinese diet, which is characterized by well-balanced dietary intake, high in fiber and low in saturated fats.[31] There may also be a possibility that the diet itself was not entirely accounting for the observed effects, but the associated whole lifestyle habits may be responsible. Patients who were conscious of a healthier dietary intake may also be more likely to adopt a healthy lifestyle habit. Although a wide range of covariates, including BMI, level of physical activity and energy intake, have been carefully adjusted in the analyses, potential residual confounding effects cannot be completely ruled out.

\section{Conclusions}

The present study suggested a potential beneficial effect on QoL for breast cancer patients following a healthy lifestyle habit. Multivariable analyses indicated that higher consumptions of vegetables and fruits were associated with better global health status/QoL and less severe symptom in diarrhea. Therefore, interventions to encourage breast cancer survivors to adopt a healthy dietary habit should be pursued. Furthermore, prospective follow-up data could confirm whether specific dietary pattern has impact on outcomes of breast cancer patients.

\section{Declarations}




\section{Funding}

This work was supported by World Cancer Research Fund International (Grant Number WCRF 2010/249 and WCRF 2014/1197).

\section{Conflicts of Interest statement}

The authors declare no potential conflicts of interest.

\section{Ethics approval}

All procedures performed in studies involving human participants were in accordance with the ethical standards of the Joint CUHK-NTEC Clinical Research Ethics Committee and the KWC Research Ethics Committee of the Chinese University of Hong Kong and the Hong Kong Hospital Authority and with the 1964 Helsinki declaration and its later amendments or comparable ethical standards. Written consent was obtained from all individual participants included in the study. The study was approved by the Joint CUHK-NTEC Clinical Research Ethics Committee and the KWC Research Ethics Committee of the Chinese University of Hong Kong and the Hong Kong Hospital Authority.

\section{Consent to participate}

Written consent was obtained from all individual participants included in the study.

\section{Consent for publication}

Written consent for publication was obtained from all individual participants included in the study.

\section{Availability of data and material}

All analyzed data during the current study were presented in the main manuscript and supplementary file. The original datasets are available from the corresponding author on reasonable request.

\section{Code availability}

Not Applicable.

\section{Author Contributions}

Y.Y. Lei, S.C. Ho and W. Yeo designed the study. C. Kwok, A. Cheng, K.L. Cheung and R. Lee collected the data. Y.Y. Lei, S.C. Ho, F. Mo and W. Yeo performed the statistical analyses, interpreted the data and drafted manuscript. All authors approved the final manuscript for publication.

\section{References}


1. Overview of Hong Kong Cancer Statistics of 2018. Hong Kong Cancer Registry, Hospital Authority. http://www3.ha.org.hk/cancereg/. Accessed Jan 2021.

2. Wong, I. O., Schooling, C. M., Cowling, B. J., \& Leung, G. M. (2015). Breast cancer incidence and mortality in a transitioning Chinese population: current and future trends. $\mathrm{Br} \mathrm{J}$ Cancer, 112(1), 167170.

3. Gong, X. H., Wang, J. W., Li, J., Chen, X. F., Sun, L., Yuan, Z. P., \& Yu, J. M. (2017). Physical exercise, vegetable and fruit intake and health-related quality of life in Chinese breast cancer survivors: a cross-sectional study. Qual Life Res, 26(6), 1541-1550.

4. Blanchard, C. M., Courneya, K. S., Stein, K., \& American Cancer Society's, S. C. S., II. (2008). Cancer survivors' adherence to lifestyle behavior recommendations and associations with health-related quality of life: results from the American Cancer Society's SCS-II. J Clin Oncol, 26(13), 2198-2204.

5. Hu, F. B. (2002). Dietary pattern analysis: a new direction in nutritional epidemiology. Curr Opin Lipidol, 13(1), 3-9.

6. Hu, F. B., Rimm, E., Smith-Warner, S. A., Feskanich, D., Stampfer, M. J., Ascherio, A., Sampson, L., \& Willett, W. C. (1999). Reproducibility and validity of dietary patterns assessed with a food-frequency questionnaire. Am J Clin Nutr, 69(2), 243-249.

7. Kroenke, C. H., Fung, T. T., Hu, F. B., \& Holmes, M. D. (2005). Dietary patterns and survival after breast cancer diagnosis. J Clin Oncol, 23(36), 9295-9303.

8. Kwan, M. L., Weltzien, E., Kushi, L. H., Castillo, A., Slattery, M. L., \& Caan, B. J. (2009). Dietary patterns and breast cancer recurrence and survival among women with early-stage breast cancer. $\mathrm{J}$ Clin Oncol, 27(6), 919-926.

9. Vrieling, A., Buck, K., Seibold, P., Heinz, J., Obi, N., Flesch-Janys, D., \& Chang-Claude, J. (2013). Dietary patterns and survival in German postmenopausal breast cancer survivors. Br J Cancer, 108(1), 188192.

10. Song, S. H., Eunkyung Moon, Hyeong-Gon Noh, Dong-Young Lee, Jung Eun (2015). Dietary patterns and health-related quality of life among Korean breast cancer survivors. The FASEB Journal, Abstract Number: 736.733.

11. Lei, Y. Y., Ho, S. C., Cheng, A., Kwok, C., Cheung, K. L., He, Y. Q., Lee, C. I., Lee, R., \& Yeo, W. (2018). Dietary changes in the first 3 years after breast cancer diagnosis: a prospective Chinese breast cancer cohort study. Cancer Manag Res, 10, 4073-4084.

12. Lei, Y. Y., Ho, S. C., Cheng, A., Kwok, C., Lee, C. I., Cheung, K. L., Lee, R., Loong, H. H. F., He, Y. Q., \& Yeo, W. (2018). Adherence to the World Cancer Research Fund/American Institute for Cancer Research Guideline Is Associated With Better Health-Related Quality of Life Among Chinese Patients With Breast Cancer. J Natl Compr Canc Netw, 16(3), 275-285.

13. Lei, Y. Y., Ho, S. C., Cheng, A., Kwok, C., Cheung, K. L., He, Y. Q., Lee, R., \& Yeo, W. (2020). The association between soy isoflavone intake and menopausal symptoms after breast cancer diagnosis: a prospective longitudinal cohort study on Chinese breast cancer patients. Breast Cancer Res Treat, 181(1), 167-180. 
14. Lei, Y.-Y., Ho, S. C., Kwok, C., Cheng, A., Cheung, K. L., Lee, R., \& Yeo, W. (2020). Longitudinal changes in sports activity from pre-diagnosis to first five years post-diagnosis: a prospective Chinese breast cancer cohort study. BMC Cancer, 20(1), 1013.

15. Edge SB, B. D., Compton CC, Fritz AG, Greene FL, Trotti A, editors. AJCC cancer staging manual (7th ed). New York, NY: Springer; 2010.

16. WHO/IASO/IOTF. The Asia-Pacific Perspective: Redefining Obesity and Its Treatment. Health Communications Australia: Melbourne, Australia, 2000.

17. Zhang, C. X., \& Ho, S. C. (2009). Validity and reproducibility of a food frequency Questionnaire among Chinese women in Guangdong province. Asia Pac J Clin Nutr, 18(2), 240-250.

18. Yang., Y.-X., Wang., G.-Y., \& Pan., X.-C. China Food Composition 2002. Beijing, China: Peking University Medical Press; 2002.

19. Dianatinasab, M., Rezaian, M., HaghighatNezad, E., Bagheri-Hosseinabadi, Z., Amanat, S., Rezaeian, S., Masoudi, A., \& Ghiasvand, R. (2020). Dietary Patterns and Risk of Invasive Ductal and Lobular Breast Carcinomas: A Systematic Review and Meta-analysis. Clin Breast Cancer, 20(4), e516-e528.

20. Chie, W. C., Chang, K. J., Huang, C. S., \& Kuo, W. H. (2003). Quality of life of breast cancer patients in Taiwan: validation of the Taiwan Chinese version of the EORTC QLQ-C30 and EORTC QLQ-BR23. Psychooncology, 12(7), 729-735.

21. Aaronson, N. K., Ahmedzai, S., Bergman, B., Bullinger, M., Cull, A., Duez, N. J., Filiberti, A., Flechtner, H., Fleishman, S. B., de Haes, J. C., \& et al. (1993). The European Organization for Research and Treatment of Cancer QLQ-C30: a quality-of-life instrument for use in international clinical trials in oncology. J Natl Cancer Inst, 85(5), 365-376.

22. Fayers, P. M., Aaronson, N. K., Bjordal, K., Groenvold, M., Curran, D., Bottomley, A., \& Group., o. b. o. t. E. Q. o. L. The EORTC QLQ-C30 scoring manual (3rd Edition). Published by: European Organisation for Research and Treatment of Cancer, Brussel 2001.

23. Kim, N. H., Song, S., Jung, S. Y., Lee, E., Kim, Z., Moon, H. G., Noh, D. Y., \& Lee, J. E. (2018). Dietary pattern and health-related quality of life among breast cancer survivors. BMC Womens Health, 18(1), 65.

24. Wayne, S. J., Baumgartner, K., Baumgartner, R. N., Bernstein, L., Bowen, D. J., \& Ballard-Barbash, R. (2006). Diet quality is directly associated with quality of life in breast cancer survivors. Breast Cancer Res Treat, 96(3), 227-232.

25. George, S. M., Alfano, C. M., Neuhouser, M. L., Smith, A. W., Baumgartner, R. N., Baumgartner, K. B., Bernstein, L., \& Ballard-Barbash, R. (2014). Better postdiagnosis diet quality is associated with less cancer-related fatigue in breast cancer survivors. J Cancer Surviv, 8(4), 680-687.

26. Mosher, C. E., Sloane, R., Morey, M. C., Snyder, D. C., Cohen, H. J., Miller, P. E., \& Demark-Wahnefried, W. (2009). Associations between lifestyle factors and quality of life among older long-term breast, prostate, and colorectal cancer survivors. Cancer, 115(17), 4001-4009.

27. Butt, Z., Rao, A. V., Lai, J.-S., Abernethy, A. P., Rosenbloom, S. K., \& Cella, D. (2010). Age-associated differences in fatigue among patients with cancer. Journal of pain and symptom management, 
40(2), 217-223.

28. Huijnen, B., van der Horst, F., van Amelsvoort, L., Wesseling, G., Lansbergen, M., Aarts, P., Nicolson, N., \& Knottnerus, A. (2005). Dyspnea in elderly family practice patients. Occurrence, severity, quality of life and mortality over an 8-year period. Family Practice, 23(1), 34-39.

29. McRorie, J. W., Jr. (2015). Evidence-Based Approach to Fiber Supplements and Clinically Meaningful Health Benefits, Part 2: What to Look for and How to Recommend an Effective Fiber Therapy. Nutr Today, 50(2), 90-97.

30. Martinez, M. E., Marshall, J. R., \& Sechrest, L. (1998). Invited commentary: Factor analysis and the search for objectivity. Am J Epidemiol, 148(1), 17-19.

31. Lv, N., \& Cason, K. L. (2004). Dietary pattern change and acculturation of Chinese Americans in Pennsylvania. J Am Diet Assoc, 104(5), 771-778.

\section{Tables}

Table 1. Representative food groups ${ }^{\mathrm{a}}$ for the two major dietary patterns identified by principal component analysis at 18-month follow-up

\begin{tabular}{|lll|}
\hline Foods/Food groups & $\begin{array}{l}\text { Factor } 1 \\
\text { Grain and animal food pattern }\end{array}$ & $\begin{array}{l}\text { Factor } 2 \\
\text { Vegetable and fruit pattern }\end{array}$ \\
\hline Refined grain & 0.722 & \\
\hline Red meat & 0.478 & \\
\hline Fish and seafood & 0.411 & \\
\hline Oil & 0.408 & \\
\hline Cakes and snacks & 0.302 & \\
\hline Processed meat & 0.266 & 0.932 \\
\hline Eggs & 0.262 & 0.489 \\
\hline Leaf vegetables & & 0.390 \\
\hline Other vegetables & & 0.314 \\
\hline Fruits & & 0.281 \\
\hline Potato & & 12.154 \\
\hline Legumes & & \\
\hline Variance explained (\%) & 26.228 & \\
\hline
\end{tabular}

${ }^{a}$ Representative food groups were selected based on factor loadings $>0.25$. 
Table 2. Characteristics of patients according to the tertiles of dietary patterns score at 18-month followup 


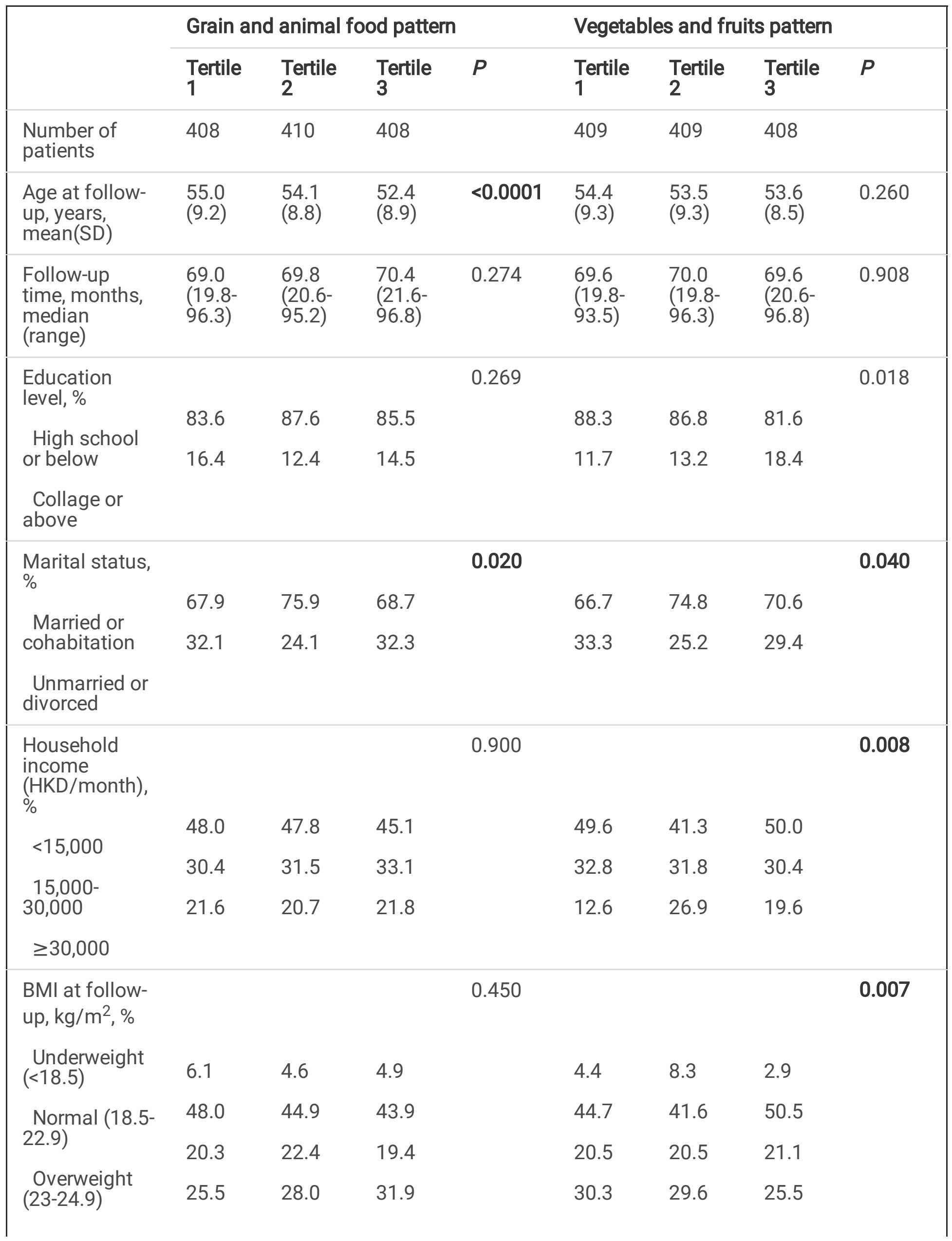




\begin{tabular}{|c|c|c|c|c|c|c|c|c|}
\hline $\begin{array}{l}\text { Number of } \\
\text { comorbidities, } \\
\%\end{array}$ & & & & 0.056 & & & & 0.094 \\
\hline None & 57.8 & 60.5 & 64.7 & & 57.9 & 62.3 & 62.7 & \\
\hline 1 & 25.5 & 26.8 & 25.5 & & 25.9 & 24.4 & 27.5 & \\
\hline$\geq 2$ & 16.7 & 12.7 & 9.8 & & 16.1 & 13.3 & 9.8 & \\
\hline Parity, \% & & & & 0.916 & & & & 0.212 \\
\hline 0 & 22.1 & 20.7 & 24.3 & & 22.2 & 19.5 & 25.2 & \\
\hline 1 & 22.8 & 22.7 & 23.0 & & 22.0 & 25.2 & 21.3 & \\
\hline 2 & 36.8 & 39.0 & 35.8 & & 35.8 & 37.2 & 38.8 & \\
\hline$\geq 3$ & 18.4 & 17.6 & 16.9 & & 20.0 & 18.1 & 14.7 & \\
\hline $\begin{array}{l}\text { Menopause } \\
\text { status at } \\
\text { follow-up, \% }\end{array}$ & & & & 0.016 & & & & 0.609 \\
\hline Pre- & 18.4 & 16.6 & 24.3 & & 79.5 & 79.5 & 81.9 & \\
\hline $\begin{array}{l}\text { Post- } \\
\text { menopausal }\end{array}$ & 81.6 & 83.4 & 75.7 & & 20.5 & 20.5 & 18.1 & \\
\hline AJCC stage at & & & & 0.056 & & & & 0.533 \\
\hline 1 & 33.1 & 30.2 & 31.4 & & 30.1 & 32.5 & 32.1 & \\
\hline 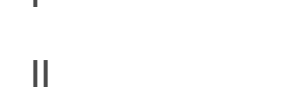 & 51.4 & 48.8 & 44.4 & & 50.6 & 46.2 & 47.8 & \\
\hline 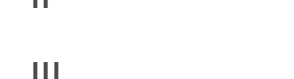 & 15.5 & 20.3 & 24.0 & & 18.6 & 21.3 & 19.4 & \\
\hline $\begin{array}{l}\text { I-III without } \\
\text { detail }\end{array}$ & 0.5 & 0.7 & 0.2 & & 0.7 & 0 & 0.7 & \\
\hline Histology, \% & & & & 0.668 & & & & 0.798 \\
\hline IDC & 90.0 & 90.0 & 89.0 & & 89.0 & 90.5 & 89.5 & \\
\hline ILC & 2.2 & 3.7 & 3.4 & & 3.9 & 2.7 & 2.7 & \\
\hline Others & 7.8 & 6.3 & 7.6 & & 7.1 & 6.8 & 7.8 & \\
\hline ER status, \% & & & & 0.204 & & & & 0.036 \\
\hline Positive & 75.2 & 74.6 & 70.9 & & 71.6 & 77.2 & 71.8 & \\
\hline Negative & 23.3 & 22.9 & 25.2 & & 24.2 & 21.8 & 25.5 & \\
\hline Missing & 1.5 & 2.4 & 3.9 & & 4.2 & 1.0 & 2.7 & \\
\hline
\end{tabular}




\begin{tabular}{|c|c|c|c|c|c|c|c|c|}
\hline PR status, $\%$ & & & & 0.397 & & & & 0.015 \\
\hline Positive & 56.6 & 59.1 & 53.7 & & 55.7 & 57.9 & 55.6 & \\
\hline Negative & 41.2 & 38.0 & 42.4 & & 39.1 & 41.1 & 41.4 & \\
\hline Missing & 2.2 & 2.9 & 3.9 & & 5.1 & 1.0 & 2.9 & \\
\hline HER2 status, & & & & 0.479 & & & & 0.579 \\
\hline & 29.9 & 27.1 & 24.5 & & 25.7 & 29.1 & 26.7 & \\
\hline positive & 66.9 & 70.0 & 71.6 & & 70.4 & 67.2 & 70.8 & \\
\hline Missing & 3.2 & 2.9 & 3.9 & & 3.9 & 3.7 & 2.5 & \\
\hline Type of & & & & 0.949 & & & & 0.596 \\
\hline surgety & 65.4 & 64.4 & 64.7 & & 66.7 & 64.3 & 63.5 & \\
\hline Conservation & 34.6 & 35.6 & 35.3 & & 33.3 & 35.7 & 36.5 & \\
\hline Chemotherapy, & & & & 0.062 & & & & 0.666 \\
\hline & 77.5 & 83.7 & 82.1 & & 82.4 & 80.0 & 80.9 & \\
\hline No & 22.5 & 16.3 & 17.9 & & 17.6 & 20.0 & 19.1 & \\
\hline Radiation & & & & 0.141 & & & & 0.476 \\
\hline (1) & 66.9 & 72.2 & 72.5 & & 71.1 & 72.1 & 68.4 & \\
\hline & 33.1 & 27.8 & 27.5 & & 28.9 & 27.9 & 31.6 & \\
\hline Hormone & & & & 0.108 & & & & 0.579 \\
\hline 19. & 77.9 & 72.9 & 78.7 & & 76.0 & 78.2 & 75.2 & \\
\hline No & 22.1 & 27.1 & 21.3 & & 24.0 & 21.8 & 24.8 & \\
\hline Physical & & & & 0.314 & & & & $<0.0001$ \\
\hline MET- & 10.9 & 10.0 & 9.5 & & 7.5 & 10.0 & 13.0 & \\
\hline mean (SD) & $(12.8)$ & (13.6) & $(13.6)$ & & $(11.4)$ & $(12.2)$ & (15.5) & \\
\hline Energy intake, & 1083.7 & 1314.7 & 1686.8 & $<0.0001$ & 1155.1 & 1354.7 & 1575.7 & $<0.0001$ \\
\hline mean (SD) & (252.5) & $(251.2)$ & (389.2) & & & (355.1) & & \\
\hline
\end{tabular}

Bold face: $P<0.05$ 
Abbreviations: SD, standard deviation; HKD, Hong Kong dollars; BMI, body mass index; AJCC, American joint Committee on cancer; IDC, invasive ductal carcinoma; ILC, invasive lobular carcinoma; DCIS, ductal carcinoma in situ; ER, estrogen receptor; PR, progesterone receptor; HER 2, human epidermal-growthfactor receptor 2; MET, metabolic equivalent of task.

Table 3. Multivariate linear regression analyses of the factor scores of "grain and animal food pattern" on QoL in Chinese women with early stage breast cancer

\begin{tabular}{|lllllll|}
\hline EORTC QLQ-C30 & \multicolumn{2}{l}{ Univariate analysis } & Model 1 & \multicolumn{3}{c|}{ Model 2 } \\
\cline { 2 - 7 } & $\boldsymbol{\beta}$ & $\boldsymbol{P}$ & $\boldsymbol{\beta}$ & $\boldsymbol{P}$ & $\boldsymbol{\beta}$ & $\boldsymbol{P}$ \\
\hline Global Health status/QoL & -0.032 & 0.262 & -0.025 & 0.551 & 0.062 & 0.226 \\
\hline Functioning & & & & & & \\
\hline Physical Functioning & -0.006 & 0.831 & 0.025 & 0.555 & 0.069 & 0.176 \\
\hline Role Functioning & -0.072 & 0.012 & -0.042 & 0.326 & -0.021 & 0.682 \\
\hline Emotional Functioning & -0.021 & 0.466 & 0.001 & 0.978 & 0.037 & 0.472 \\
\hline Cognitive Functioning & 0.006 & 0.822 & 0.058 & 0.180 & 0.085 & 0.104 \\
\hline Social Functioning & -0.042 & 0.141 & -0.008 & 0.855 & -0.017 & 0.745 \\
\hline Symptoms & & & & & & \\
\hline Fatigue & 0.022 & 0.440 & -0.086 & 0.044 & -0.133 & 0.010 \\
\hline Nausea and vomiting & 0.054 & 0.057 & 0.019 & 0.654 & 0.002 & 0.974 \\
\hline Pain & 0.040 & 0.163 & -0.033 & 0.444 & -0.058 & 0.261 \\
\hline Dyspnea & 0.015 & 0.608 & -0.082 & 0.056 & -0.117 & $\mathbf{0} .024$ \\
\hline Insomnia & 0.013 & 0.643 & -0.011 & 0.803 & -0.032 & 0.540 \\
\hline Loss of appetite & 0.008 & 0.783 & -0.008 & 0.847 & -0.068 & 0.193 \\
\hline Constipation & 0.017 & 0.546 & 0.041 & 0.347 & -0.007 & 0.899 \\
\hline Diarrhea & 0.049 & 0.084 & 0.062 & 0.144 & -0.012 & 0.810 \\
\hline Financial impact & 0.055 & 0.054 & -0.055 & 0.192 & -0.055 & 0.281 \\
\hline
\end{tabular}

Bold face: $P<0.05$

Multivariate linear regressions were used to analyze the association of "grain and animal food pattern" and QoL. QoL items were $\log _{10}$ transformed. The potential confounders were introduced in the models using the enter method. Model 1, adjusted for age at 18-month follow-up, education level, income status, total number of comorbidities, menopausal status at 18-month follow-up, AJCC stage, ER status and PR 
status, current adjuvant hormonal therapy usage, BMI at 18-month follow-up, level of physical activity, total energy intake; Model 2, further adjusted for factor scores of "vegetables and fruits pattern".

Abbreviations: $\beta$, standardized coefficient of linear regression model; QoL, quality of life; EORTC QLQ-C30, European Organization for Research and Treatment of Cancer Quality of Life Questionnaire Core 30; AJCC, American joint Committee on cancer; ER, estrogen receptor; PR, progesterone receptor.

Table 4. Multivariate linear regression analyses of the factor score of "vegetables and fruits pattern" on QoL in Chinese women with early stage breast cancer

\begin{tabular}{|lllllll|}
\hline EORTC QLQ-C30 & \multicolumn{2}{l}{ Univariate analysis } & Model 1 & \multicolumn{3}{c|}{ Model 2 } \\
\cline { 2 - 7 } & $\boldsymbol{\beta}$ & $\boldsymbol{P}$ & $\boldsymbol{\beta}$ & $\boldsymbol{P}$ & $\boldsymbol{\beta}$ & $\boldsymbol{P}$ \\
\hline Global Health status/QoL & 0.084 & 0.003 & 0.092 & 0.004 & 0.118 & $\mathbf{0 . 0 0 2}$ \\
\hline Functioning & & & & & & \\
\hline Physical Functioning & 0.043 & 0.132 & 0.031 & 0.337 & 0.060 & 0.121 \\
\hline Role Functioning & 0.008 & 0.790 & 0.038 & 0.249 & 0.029 & 0.465 \\
\hline Emotional Functioning & 0.026 & 0.363 & 0.033 & 0.305 & 0.049 & 0.210 \\
\hline Cognitive Functioning & -0.005 & 0.865 & 0.001 & 0.983 & 0.036 & 0.357 \\
\hline Social Functioning & -0.028 & 0.327 & -0.005 & 0.873 & -0.012 & 0.754 \\
\hline Symptoms & & & & & & \\
\hline Fatigue & 0.005 & 0.873 & -0.008 & 0.815 & -0.064 & 0.102 \\
\hline Nausea and vomiting & -0.019 & 0.517 & -0.025 & 0.449 & -0.024 & 0.542 \\
\hline Pain & 0.004 & 0.892 & -0.010 & 0.763 & -0.034 & 0.381 \\
\hline Dyspnea & 0.012 & 0.683 & 0.002 & 0.959 & -0.048 & 0.224 \\
\hline Insomnia & -0.011 & 0.699 & -0.015 & 0.641 & -0.029 & 0.467 \\
\hline Loss of appetite & -0.052 & 0.068 & -0.052 & 0.110 & -0.081 & $\mathbf{0 . 0 4 0}$ \\
\hline Constipation & -0.055 & 0.054 & -0.062 & 0.060 & -0.065 & 0.102 \\
\hline Diarrhea & -0.082 & 0.004 & -0.097 & 0.003 & -0.102 & $\mathbf{0 . 0 0 9}$ \\
\hline Financial impact & 0.062 & 0.031 & 0.023 & 0.463 & 0.001 & 0.992 \\
\hline
\end{tabular}

Bold face: $P<0.05$

Multivariate linear regressions were used to analyze the association of "vegetables and fruits pattern" and QoL. QoL items were $\log _{10}$ transformed. The potential confounders were introduced in the models using 
the enter method. Model 1, adjusted for age at 18-month follow-up, education level, income status, total number of comorbidities, menopausal status at 18-month follow-up, AJCC stage, ER status and PR status, current adjuvant hormonal therapy usage, BMI at 18-month follow-up, level of physical activity, total energy intake; Model 2, further adjusted for factor scores of "grain and animal food pattern".

Abbreviations: $\beta$, standardized coefficient of linear regression model; QoL, quality of life; EORTC QLQ-C30, European Organization for Research and Treatment of Cancer Quality of Life Questionnaire Core 30; AJCC, American joint Committee on cancer; ER, estrogen receptor; PR, progesterone receptor.

\section{Supplementary Files}

This is a list of supplementary files associated with this preprint. Click to download.

- Supplementarymaterials.docx 